\title{
AN EMBEDDING THEOREM FOR COMPACT SEMILATTICES ${ }^{1}$
}

\author{
J. W. LEA, JR.
}

\begin{abstract}
It is shown that if a compact topological semilattice $S$ is a topological lattice, then $S$ can be embedded simultaneously algebraically and topologically in a direct product of $n$ chains if and only if $S$ can be algebraically embedded in a direct product of $n$ chains.
\end{abstract}

K. A. Baker and A. R. Stralka [1] proved that a compact distributive topological lattice of finite breadth can be embedded algebraically and topologically in a finite direct product of compact chains. The purpose of this paper is to obtain a similar result for compact topological semilattices. The primary difficulty in adapting the method of Baker and Stralka was proving the continuity of the embedding.

The author wishes to express his gratitude to Professor J. D. Lawson for his encouragement and advice during the preparation of this paper.

1. Preliminaries. If $S$ is a partially ordered set and every two elements $x, y$ have a greatest lower bound $x \wedge y$, then $S$ is a (meet) semilattice; if every two elements $x, y$ also have a least upper bound $x \vee y$, then $S$ is a lattice. If $S$ is also a Hausdorff topological space and $\bigwedge: S \times S \rightarrow S$ is continuous, then $S$ is a topological semilattice; if $S$ is a topological semilattice with respect to both $\bigwedge$ and $\bigvee$, then $S$ is a topological lattice. The breadth of a lattice is the smallest integer $n$ such that any meet $x_{1} \wedge \cdots \wedge x_{m}(m>n)$ is a meet of a subset of $n$ of the $x_{i}$. The width $w(X)$ of a partially ordered set $X$ is the maximum number of elements in a set of incomparable elements.

If $S$ is a semilattice and $x \in S$, then $M(x)=\{y \in S: x \leqq y\} ; L(x)$ is defined dually; if $x \leqq y$, then $[x, y]=M(x) \cap L(y)$. An element $x$ in a semilattice $S$ is meet irreducible if $a, b \in S$ and $x=a \wedge b$ imply $x=a$ or $x=b$. We shall denote by $\operatorname{MI}(S)$ the set of all meet irreducible elements of $S$.

Let $A^{*}$ and $A^{\circ}$ denote, respectively, the closure and interior of $A$.

Presented to the Society, November 20, 1971; received by the editors August 2, 1971. AMS 1970 subject classifications. Primary 06A20, 54F05, 22A30.

Key words and phrases. Compact semilattice, compact lattice, direct product of chains, meet irreducible.

${ }^{1}$ This paper consists of part of a dissertation presented to the Graduate School of the Louisiana State University.

(c) American Mathematical Society 1972 


\section{The embedding theorem.}

Definition 2.1. If $S$ is a topological semilattice and $x \in S$, then $x$ is a local maximum of $S$ if $\{x\}$ is open in $M(x)$. If $C$ is a chain in $S$, then $x$ is a local maximum of $C$, i.e., $\{x\}$ is open in $M(x) \cap C$, when $x \neq \wedge D$ for any $D \subset C \backslash\{x\}$.

Definition 2.2. Let $L$ be a lattice with 1 and $C$ a chain in $L$ which contains 1 and which is closed under arbitrary meets in $L$, i.e., $A \subset C$ implies $\bigwedge A$ exists in $L$ and $\bigwedge A \in C$. Then $\sigma: L \rightarrow C$ is defined by $\sigma(x)=$ $\bigwedge\{p \in C: x \leqq p\}$ for all $x \in L$.

In [1] it is shown that $\sigma$ is order preserving, $x \leqq \sigma(x)$ for all $x \in L$, $\sigma(p)=p$ for all $p \in C$, and $\sigma(x \vee y)=\sigma(x) \vee \sigma(y)$ for all $x, y \in L$.

The interval topology on a partially ordered set $X$ with 0 and 1 is defined by taking $\{[a, b]: a \leqq b, a, b \in X\}$ as a subbasis for the closed sets. If $L$ is a lattice with 1 and $C$ is a chain in $L$ which contains 1 and which is closed under arbitrary meets in $L$, then $C$ is a complete lattice [2] and $C$ is a compact topological lattice in its interval topology [5].

LEMMA 2.3. Let $L$ be a compact topological lattice of finite breadth and let $C$ be a chain in $L$ such that:

(i) $1 \in C$ and $C$ is closed under arbitrary meets in $L$;

(ii) $x$ a local maximum of $C$ implies $x$ a local maximum of $L$;

(iii) $x, y \in C$ and $x<y$ imply $x \in L(y)^{\circ}$.

Then $\sigma: L \rightarrow C$ is a continuous join homomorphism when $C$ has the interval topology.

Proof. Let $z=\wedge C$. Because $C$ has the interval topology we need only show that $\sigma^{-1}([z, p) \cap C)$ is open and $\sigma^{-1}([z, p] \cap C)$ is closed for all $p \in C$. Let $W=\sigma^{-1}([z, p) \cap C)$ and let $x \in W$. Then $\sigma(x)<p$; thus $\sigma(x) \in$ $L(p)^{\circ}$. We consider two cases.

Case 1. Suppose $\sigma(x)$ is a local maximum of $C$. Then $\sigma(x)$ is a local maximum of $L$; hence $\{\sigma(x)\}$ is open in $M(\sigma(x))$. The function $f: L \rightarrow$ $M(\sigma(x))$ defined by $f(y)=\sigma(x) \vee y$ for all $y \in L$ is continuous. Thus $L(\sigma(x))=f^{-1}(\sigma(x))$ is open in $L ; x \in L(\sigma(x))$. Clearly $L(\sigma(x)) \subset W$.

Case 2. Suppose $\sigma(x)$ is not a local maximum of $C$. Then $\{\sigma(x)\}$ is not open in $M(\sigma(x)) \cap C$. Thus there is an $s \in(M(\sigma(x)) \cap C) \cap([z, p) \cap C)$ such that $s \neq \sigma(x)$. Hence $\sigma(x)<s<p$; therefore $\sigma(x) \in L(s)^{\circ}$. Therefore since $x \leqq \sigma(x), x \in L(s)^{\circ}$. Clearly $\sigma\left(L(s)^{\circ}\right) \subset[z, s] \cap C$. Hence $x \in L(s)^{\circ} \subset W$. Since $x$ was arbitrary, $W$ is open.

Let $F=\sigma^{-1}([z, p] \cap C)$. Thus $F=L(p)$ which is closed. Hence we have shown that $\sigma$ is continuous. As remarked above $\sigma$ is a join homomorphism.

Suppose $L$ is a compact lattice of finite breadth $n$. Each $x \in L$ can be written as the meet of at most $n$ meet irreducibles [1]. If $w(\mathrm{MI}(L))$ is less than $n$, then each $x \in L$ can actually be written as a meet of fewer than $n$ 
meet irreducibles. It follows that any finite meet-irredundant set contains less than $n$ elements contrary to the assumption that $L$ has breadth $n$. We have proved:

Proposition 2.4. If $L$ is a compact topological lattice of finite breadth $n$, then $w(\mathrm{MI}(L)) \geqq n$.

The next definition is a generalization of a notion due to Baker and Stralka [1].

Definition 2.5. If $L$ is a lattice with 1 and $C$ is a chain in $L$, then $C$ is a coordinate chain of $L$ if

(a) $1 \in C$,

(b) $C$ is closed under arbitrary meets in $L$,

(c) $x \in C$ implies $x=\wedge D$ for some $D \subset \mathrm{MI}(L) \cap C$.

LEMMA 2.6. Let $L$ be a compact topological lattice of finite breadth for which $w(\mathrm{MI}(L))=n$. Then there exist coordinate chains $C_{1}, \cdots, C_{n}$ of $L$ such that

(i) $\mathrm{MI}(L) \subset \bigcup_{i=1}^{n} C_{i}$,

(ii) $x \in L$ implies $x=\bigwedge_{i=1}^{n} \sigma_{i}(x)$ where $\sigma_{i}: L \rightarrow C_{i}$ for $i=1, \cdots, n$,

(iii) $p$ a local maximum of $C_{i}$ implies $p$ a local maximum of $L$ for $i=1, \cdots, n$,

(iv) $x, y \in C_{i}$ and $x<y$ imply $x \in L(y)^{\circ}$ for $i=1, \cdots, n$.

Proof. Let $\mathscr{S}$ be the set of all $\left(C_{1}, \cdots, C_{n}\right)$ such that for each $i$ between 1 and $n$, (1) $C_{i}$ is a chain in $L$, (2) $1 \in C_{i}$, (3) $x \in \operatorname{MI}(L)$ and $x=$ $\bigwedge D$ for some $D \subset C_{i}$ implies $x \in C_{i}$, and (4) $\operatorname{MI}(L)=\bigcup_{i=1}^{n} C_{i}$. Since $w(\mathrm{MI}(L))=n$ we can find chains $A_{1}, \cdots, A_{n}$ such that $\mathrm{MI}(L)=\bigcup_{i=1}^{n} A_{i}[3]$. For each $i=1, \cdots, n$, let $C_{i}^{\prime}=\{1\} \cup\left\{x \in \operatorname{MI}(L): x=\bigwedge D\right.$ for some $\left.D \subset A_{i}\right\}$. Since $C_{i}^{\prime} \subset A_{i}^{*} \cup\{1\}, C_{i}^{\prime}$ is a chain $; 1 \in C_{i}^{\prime} ; \operatorname{MI}(L)=\bigcup_{i=1}^{n} C_{i}^{\prime}$. Thus to conclude that $\left(C_{1}^{\prime}, \cdots, C_{n}^{\prime}\right) \in \mathscr{S}$, we need to show that $D \subset C_{i}^{\prime}$ and $\wedge D \in \operatorname{MI}(L)$ imply $\wedge D \in C_{i}^{\prime}$ for $i=1, \cdots, n$. Let $D \subset C_{i}^{\prime}$ and suppose $\wedge D \in \operatorname{MI}(L)$. Each $x \in D$ belongs to $A_{i}$ or is equal to $\bigwedge D_{x}$ for some $D_{x} \subset A_{i}$. Let $E=\left(\bigcup\left\{D_{x}: x \in D \backslash A_{i}\right\}\right) \cup\left(D \cap A_{i}\right)$. Then $E \subset A_{i}$ and $\wedge E=\wedge D$; hence $\wedge D \in C_{i}^{\prime}$. Thus $\mathscr{S} \neq \varnothing$.

We partially order $\mathscr{S}$ by coordinatewise set inclusion. Let

$$
\left.\left\{C_{1 \alpha}, \cdots, C_{n \alpha}\right)\right\}_{\alpha \in \Delta}
$$

be a chain in $\mathscr{S}$. Let $C_{i}^{\prime \prime}=\bigcap_{\alpha \in \Delta} C_{i \alpha}$ for $i=1, \cdots, n$. Clearly each $C_{i}^{\prime \prime}$ is a chain, contains 1 , and contains $\wedge E$ whenever $E \subset C_{i}^{\prime \prime}$ and $\wedge E \in \operatorname{MI}(L)$. By standard finiteness arguments we see that $\operatorname{MI}(L)=\bigcup_{i=1}^{n} C_{i}^{\prime \prime}$. Therefore $\left(C_{1}^{\prime \prime}, \cdots, C_{n}^{\prime \prime}\right) \in \mathscr{S}$. Thus any chain in $\mathscr{S}$ has a lower bound in $\mathscr{S}$; therefore by Zorn's lemma $\mathscr{S}$ must contain a minimal element, say $\left(M_{1}, \cdots, M_{n}\right)$. 
Let $C_{i}$ be the meet closure of $M_{i}$ for $i=1, \cdots, n$. It follows that $C_{1}, \cdots, C_{n}$ are coordinate chains of $L$ which satisfy part (i). In [1] it is shown that any element of a compact topological lattice of finite breadth $n$ is the meet of $n$ or fewer meet irreducibles. Thus part (ii) is satisfied since by Proposition 2.4 the breadth of $L$ is less than or equal to $n$.

To prove part (iii), suppose $p$ is a local maximum of $C_{i}$; then $p \in M_{i}$, for otherwise $p=\bigwedge D$ for some $D \subset M_{i}$ and $D$ is a decreasing net since $M_{i}$ is a chain; hence $D$ converges to $\wedge D$ [6]. Now suppose that $p$ is not a local maximum of $L$. There must exist a net $\left\{x_{\alpha}\right\}_{\alpha \in \Delta}$ in $L$ such that $p<x_{\alpha}$ and $\left\{x_{\alpha}\right\}_{\alpha \in \Delta}$ converges to $p$. By part (ii) $x_{\alpha}=\bigwedge_{i=1}^{n} \sigma_{i}\left(x_{\alpha}\right)$ for all $\alpha \in \Delta$. Choosing subnets if necessary $\left\{\sigma_{i}\left(x_{\alpha}\right)\right\}_{\alpha \in \Delta}$ converges to $p_{i}$ for $i=1, \cdots, n$. Since $p \in M_{i}, p$ is meet irreducible. Therefore $p=p_{j}$ for some $j ; p_{j}=$ $\bigwedge_{\alpha \in \Delta} \sigma_{j}\left(x_{\alpha}\right)$. Since $p$ is a local maximum of $C_{i}, j \neq i$. But now

$$
\left(M_{1}, \cdots, M_{i} \mid\{p\}, \cdots, M_{n}\right) \in \mathscr{S}
$$

and is strictly less than $\left(M_{1}, \cdots, M_{n}\right)$ contrary to the minimality of $\left(M_{1}, \cdots, M_{n}\right)$. Hence $p$ must be a local maximum of $L$.

For the proof of part (iv) we need the following lemma.

LeMma 2.7. Let $S$ be a compact topological semilatice such that $S=\bigwedge_{i=1}^{n} C_{i}$, where $C_{i}$ is a chain contained in $\operatorname{MI}(S)$ for $i=1, \cdots, n$. If $x \in C_{i}, y \in C_{i}^{*}, x<y$, and for all $j \neq i$, either (1) $y \in C_{j}^{*}$ or (2) $x \neq \wedge D$ for any $D \subset C_{j} \mid\{x\}$, then $x \in L(y)^{\circ}$.

Proof. For convenience we assume $x \in C_{n}$ and $y \in C_{n}^{*}$. Suppose $x \notin L(y)^{\circ}$. Then each open set containing $x$ must meet $S \backslash L(y)$; hence there is a net $\left\{x_{\alpha}\right\}_{\alpha \in \Delta}$ converging to $x$ such that $x_{\alpha} \leqq y$ for all $\alpha \in \Delta$. By hypothesis each $x_{\alpha}=x_{\alpha 1} \wedge \cdots \wedge x_{\alpha n}$ with $x_{\alpha i} \in C_{i}$ for $i=1, \cdots, n$ and $\alpha \in \Delta$. Since each $C_{i}^{*}$ is a chain, $x_{\alpha i}$ and $y$ are related whenever $y \in C_{i}^{*}$. If $y \in C_{i}^{*}$ and $x_{\alpha i} \leqq$ $y$, then $x_{\alpha} \leqq y$ contrary to $x_{\alpha} \leq y$. Thus $y<x_{\alpha i}$ whenever $y \in C_{i}^{*}$. Choosing subnets if necessary we have $\left\{x_{\alpha i}\right\}$ converging to $x_{i} \in C_{i}^{*}$ for $i=1, \cdots, n$. Since $x_{\alpha}=x_{\alpha 1} \wedge \cdots \wedge x_{\alpha n}$ and $\left\{x_{\alpha}\right\}$ converges to $x, x=x_{1} \wedge \cdots \wedge x_{n}$. Assume the $C_{i}$ are renumbered so that $y \notin C_{i}^{*}$ for $i=1, \cdots, k-1$ with $k-1<n$.

Since $x$ is meet irreducible, $x=x_{1}$ or $x=x_{2} \wedge \cdots \wedge x_{n}$. Suppose $x=x_{1}$. If $x_{\alpha 1} \leqq x$ for any $\alpha \in \Delta$, then $x_{\alpha} \leqq x_{\alpha 1} \leqq x<y$ contrary to $x_{\alpha} \leq y$. Since $C_{1}^{*}$ is a chain, we conclude $x<x_{\alpha 1}$ for all $\alpha$. Thus $x=x_{1}=\bigwedge_{\alpha} x_{\alpha 1}$, and $y \notin C_{1}^{*}$. Thus we have contradicted the hypothesis that for $j \neq n$, either $y \in C_{j}^{*}$ or $x \neq \bigwedge D$ for any $D \subset C_{j} \mid\{x\}$. Therefore $x \neq x_{1}$. Similarly $x \neq x_{j}$ for $j=2, \cdots, k-1$. Thus $x=x_{k}$ or $x=x_{k+1} \wedge \cdots \wedge x_{n}$. As noted above, $y$ is less than $x_{\alpha k}$ for all $\alpha$ since $y \in C_{k}^{*}$; therefore $y \leqq x_{k}$. Hence if $x=x_{k}$, we have $x<y \leqq x$, a contradiction. Similarly $x \neq x_{j}$ for $j=k+1, \cdots, n$. This contradiction completes the proof. 
We shall now prove part (iv) of Lemma 2.6. Let $x, y \in C_{i}$ with $x<y$. By the way $C_{i}$ is defined, we may choose $p \in M_{i}$ such that $x \leqq p<y$. If $p \in L(y)^{\circ}$, then $x \in L(y)^{\circ}$. Therefore we may assume $x \in M_{i}$. Suppose that $x \notin L(y)^{\circ}$. By Lemma 2.7 there exists $j \neq i$ and $D \subset M_{j}$ such that $x=\bigwedge D$. Thus $x \in M_{j}$ and $x$ is not a local maximum of $M_{i}$. Then for each $s \in M_{i}$ such that $x<s<y, s \notin L(y)^{\circ}$ since $s \in L(y)^{\circ}$ implies $x \in L(y)^{\circ}$. Thus by Lemma 2.7, for each $s \in M_{i}$ such that $x<s<y$ there exists $k \neq i$ such that $s \in M_{k}$. Therefore $\left(M_{1}, \cdots,\left(M_{i} \cap[y, 1]\right) \cup\left(M_{i} \cap L(x)\right), \cdots, M_{n}\right) \in \mathscr{S}$ contrary to the minimality of $\left(M_{1}, \cdots, M_{n}\right)$. We conclude that $x \in L(y)^{\circ}$.

THEOREM 2.8. Let $L$ be a compact topological lattice of finite breadth. If $w(\mathrm{MI}(L))=n$, then $L$ can be embedded in a direct product of $n$ compact chains by a join preserving homeomorphism.

Proof. Let $C_{1}, \cdots, C_{n}$ be the $n$ coordinate chains constructed in Lemma 2.6. Each of these chains is a compact topological lattice when given the interval topology. By Lemma 2.3, $\sigma_{i}: L \rightarrow C_{i}$ is a continuous join homomorphism for $i=1, \cdots, n$. Thus $f: L \rightarrow \prod_{i=1}^{n} C_{i}$ defined by $f(x)=$ $\left(\sigma_{1}(x), \cdots, \sigma_{n}(x)\right)$ is a continuous join homomorphism. To see that $f$ is one-to-one, suppose that for some $x, y \in L, f(x)=f(y)$. Then

$$
\left(\sigma_{1}(x), \cdots, \sigma_{n}(x)\right)=\left(\sigma_{1}(y), \cdots, \sigma_{n}(y)\right)
$$

which implies $\sigma_{i}(x)=\sigma_{i}(y)$ for $i=1, \cdots, n$. Thus $x=\bigwedge_{i=1}^{n} \sigma_{i}(x)=$ $\bigwedge_{i=1}^{n} \sigma_{i}(y)=y$; hence $f$ is one-to-one. Since $L$ is compact and $\prod_{i=1}^{n} C_{i}$ is Hausdorff, $f$ is a homeomorphism.

Proposition 2.9. Let $L$ be a lattice that is a join subsemilattice of $a$ product of $n$ chains. Then $w(\mathrm{MI}(L)) \leqq n$.

Proof. Suppose not. Then we may find $n+1$ pairwise unrelated elements of $\operatorname{MI}(L)$, say $x_{0}, \cdots, x_{n}$, where $x_{i}=\left(x_{i 1}, \cdots, x_{i n}\right)$ for $i=0, \cdots, n$. Since there are only $n$ coordinates for each $x_{i}$, there must be one point, say $x_{0}$, such that for each $j=1, \cdots, n$, there is some $i$ for which $x_{0 j} \nless x_{i j}$. Let $y_{i}=x_{0} \vee x_{i}, i=1, \cdots, n$. Note that for each $j$, there exists an $i$ such that $x_{0 j}=y_{i j}$. Then $y_{i} \neq x_{0}$, since $x_{i}$ and $x_{0}$ are unrelated, but $y_{1} \wedge \cdots \wedge y_{n}=$ $x_{0}$. This contradicts the meet irreducibility of $x_{0}$. Thus $w(\operatorname{MI}(L)) \leqq n$.

As an immediate consequence we may state:

COROLLARY 2.10. Let L be a compact topological lattice of finite breadth. Then $w(\mathrm{MI}(L))=n$ if and only if $n$ is the least number of chains in a direct product of which $L$ can be embedded as a join subsemilattice.

We are now able to obtain the embedding theorem for compact semilattices. 
THEOREM 2.11. If a compact topological semilattice $S$ is a topological lattice, then $S$ can be embedded simultaneously algebraically and topologically in a direct product of $n$ chains if and only if $S$ can be algebraically embedded in a direct product of $n$ chains.

Proof. If $S$ can be embedded algebraically in a direct product of $n$ chains then by Proposition 2.9 (or its dual) $w(\mathrm{MI}(S)) \leqq n($ or $w(\mathrm{JI}(S)) \leqq n)$ so that the hypotheses of Theorem 2.8 (or its dual) are satisfied.

3. Examples. The following example is due to D. E. Edmondson [4].

EXAMPLE 3.1. Let $C$ be a compact connected topological lattice which is a chain and let $A=\{1,2, \cdots, n\}$ with $3 \leqq n<\infty$. Let $L$ be the set of all $n$-tuples $\left(x_{1}, \cdots, x_{n}\right)$ where each $x_{i} \in C$ and where there exists $k \in A$ such that $i, j \in A \backslash\{k\}$ implies $x_{i} \leqq x_{k}$ and $x_{i}=x_{j}$. For $\left(x_{1}, \cdots, x_{n}\right)$ and $\left(y_{1}, \cdots, y_{n}\right) \in L$ define $\left(x_{1}, \cdots, x_{n}\right) \wedge\left(y_{1}, \cdots, y_{n}\right)$ to be $\left(x_{1} \wedge y_{1}, \cdots, x_{n} \wedge y_{n}\right)$; if for all $i \in A, x_{1}=x_{i}$ or $y_{1}=y_{i}$ or there is some $k \in A$ such that $x_{i} \leqq x_{k}$ and $y_{i} \leqq y_{k}$, define $\left(x_{1}, \cdots, x_{n}\right) \vee\left(y_{1}, \cdots, y_{n}\right)$ to be $\left(x_{1} \vee y_{1}, \cdots, x_{n} \vee y_{n}\right)$; if there exist $k \neq m \in A$ such that $x_{i}<x_{k}$ for all $i \in A \backslash\{k\}$ and $y_{i}<y_{m}$ for all $i \in A \backslash\{m\}$, define $\left(x_{1}, \cdots, x_{n}\right) \vee\left(y_{1}, \cdots, y_{n}\right)$ to be $\left(z_{1}, \cdots, z_{n}\right)$ where $z_{j}=x_{j} \vee y_{j}$ for $j \in\{k, m\}$ and $z_{j}=z_{k} \wedge z_{m}$ for $j \in A \backslash\{k, m\}$. With the order and topology inherited from the direct product of $n$ copies of $C, L$ is a compact connected modular topological lattice which is not distributive. Also, $L$ has breadth two and $w(\operatorname{MI}(L))=n$.

Other examples may be constructed by taking direct products of copies of these with the same or different $n$ or direct products of these with chains.

EXAMPLE 3.2. Let $I \times I$ be the unit square considered as a meet semilattice, let $A$ be the ideal $(\{0\} \times I) \cup(I \times\{0\})$, and let $S$ be the Rees quotient of $I \times I$ modulo $A$. Then $S$ is a compact connected topological semilattice of breadth two which can be algebraically embedded in $I \times I$ but cannot be topologically and algebraically embedded in any finite product of chains.

\section{REFERENCES}

1. K. A. Baker and A. R. Stralka, Compact distributive lattices of finite breadth, Pacific J. Math. 34 (1970), 311-320.

2. G. Birkhoff, Lattice theory, 3rd ed., Amer. Math. Soc. Colloq. Publ., vol. 25, Amer. Math. Soc., Providence, R.I., 1967. MR 37 \#2638.

3. R. P. Dilworth, A decomposition theorem for partially ordered sets, Ann. of Math. (2) 51 (1950), 161-166. MR 11, 309.

4. D. E. Edmondson, A modular topological lattice, Pacific J. Math. 29 (1969), 271-277. MR 39 \#4062. 
5. O. Frink, Jr., Topology in lattices, Trans. Amer. Math. Soc. 51 (1942), 569-582. MR 3, 313.

6. D. P. Strauss, Topological lattices, Proc. London Math. Soc. 18 (1968), 217-230.

Department of Mathematics, Louisiana State University, Baton Rouge, LOUISIANA 70803

Department of Mathematics, Middle Tennessee State University, MurfreesBORO, TENNESSEE 37130 